\title{
A rounding algorithm for approximating minimum Manhattan networks ${ }^{1}$
}

\author{
Victor Chepoi, Karim Nouioua, Yann Vaxès \\ Laboratoire d'Informatique Fondamentale de Marseille, \\ Université de la Méditerranée, Faculté des Sciences de \\ Luminy, F-13288 Marseille Cedex 9, France, \\ $\{$ chepoi,nouioua,vaxes\}@lif.univ-mrs.fr
}

\begin{abstract}
For a set $T$ of $n$ points (terminals) in the plane, a Manhattan network on $T$ is a network $N(T)=(V, E)$ with the property that its edges are horizontal or vertical segments connecting points in $V \supseteq T$ and for every pair of terminals, the network $N(T)$ contains a shortest $l_{1}$-path between them. A minimum Manhattan network on $T$ is a Manhattan network of minimum possible length. The problem of finding minimum Manhattan networks has been introduced by Gudmundsson, Levcopoulos, and Narasimhan (APPROX'99) and its complexity status is unknown. Several approximation algorithms (with factors 8,4, and 3) have been proposed; recently Kato, Imai, and Asano (ISAAC'02) have given a factor 2 approximation algorithm, however their correctness proof is incomplete. In this paper, we propose a rounding 2-approximation algorithm based on a LP-formulation of the minimum Manhattan network problem.
\end{abstract}

Keywords. $l_{1}$-distance, network design, linear programming, approximation algorithms.

Corresponding author: Victor Chepoi

\footnotetext{
${ }^{1}$ An extended abstract of this paper appeared in the proceedings of the 8th. International Workshop on Approximation Algorithms for Combinatorial Optimization Problems, APPROX'05, LNCS 3624, pp. 40-51.
} 


\section{Introduction}

A rectilinear path $P$ between two points $p, q$ of the plane $\mathbb{R}^{2}$ is a path connecting $p$ and $q$ and consisting of only horizontal and vertical line segments. More generally, a rectilinear network $N=(V, E)$ consists of a finite set $V$ of points of $\mathbb{R}^{2}$ (the vertices of $N$ ) and of a finite set of horizontal and vertical segments connecting pairs of points of $V$ (the edges of $N$ ). The length $l(P)($ or $l(N))$ of a rectilinear path $P$ (or of a rectilinear network $N$ ) is the sum of lengths of its edges. Analogously, the length $l(N)$ of a rectilinear network $N$ is the sum of lengths of its edges. The $l_{1}$-distance between two points $p=\left(p^{x}, p^{y}\right)$ and $q=\left(q^{x}, q^{y}\right)$ in the plane $\mathbb{R}^{2}$ is $d(p, q):=|| p-q \|_{1}=\left|p^{x}-q^{x}\right|+\left|p^{y}-q^{y}\right|$. An $l_{1}$-path between two points $p, q \in \mathbb{R}^{2}$ is a rectilinear path connecting $p, q$ and having length $d(p, q)$.

Given a set $T=\left\{t_{1}, \ldots, t_{n}\right\}$ of $n$ points (terminals) in the plane, a Manhattan network [4] on $T$ is a rectilinear network $N(T)=(V, E)$ such that $T \subseteq V$ and for every pair of points in $T$, the network $N(T)$ contains an $l_{1}$-path between them. A minimum Manhattan network on $T$ is a Manhattan network of minimum possible length and the Minimum Manhattan Network problem $(M M N$ problem $)$ is to find such a network.

The minimum Manhattan network problem has been introduced by Gudmundsson, Levcopoulos, and Narasimhan [4] and its complexity status is unknown. Gudmundsson et al. [4] proposed an $O\left(n^{3}\right)$-time algorithm with approximation factor 4 , and an $O(n \log n)$-time algorithm with approximation factor 8 . They also conjectured that there exists a 2 -approximation algorithm for this problem. Kato, Imai, and Asano [6] presented a factor 2 approximation algorithm, however, their correctness proof is incomplete. Following [6], Benkert, Shirabe, and Wolff [1] and Benkert, Wolff, and Widmann [2] described an $O(n \log n)$-time algorithm with approximation factor 3 and presented a mixed-integer programming formulation of the MMN problem. Notice that all four mentioned algorithms are geometric and some of them employ results from computational geometry. Nouioua [8] presented another $O(n \log n)$ factor 3 approximation algorithm based on the primal-dual method from linear programming. In this paper we present a rounding method applied to the optimal solution of the flow based linear program described in $[1,8]$ which leads to a factor 2 approximation algorithm for the minimum Manhattan network problem (for approximation algorithms based on rounding techniques, see the book by Vazirani [12]). For this, we define two subsets of pairs of terminals, called strips and staircases, and for each of them, we describe a specific rounding procedure. Each rounded up edge is paid by a group of parallel edges which together support at least one-half unit of fractional flow. Finally, we prove that a rectilinear network containing $l_{1}$-paths between all the pairs belonging to strips and staircases is a Manhattan network and thus, we end-up with an integer feasible solution whose cost is at most twice the fractional optimum.

Gudmundsson et al. [4] introduced the minimum Manhattan networks in connection with the construction of sparse geometric spanners. Given a set $T$ of $n$ points in the plane endowed with a norm $\|\cdot\|$, and a real number $t \geq 1$, a geometric network $N$ is a $t$-spanner for $T$ if for each pair of points $p, q \in T$, there exists a $p q$-path in $N$ of length at most $t$ times the distance $\|p-q\|$ between $p$ and $q$. In the Euclidian plane (and more generally, for $l_{p}$-planes with 


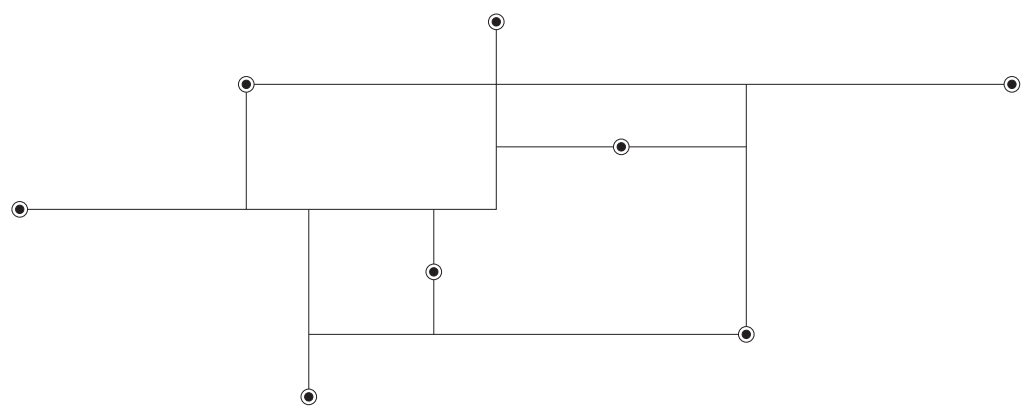

Figure 1: A minimum Manhattan network

$p \geq 2$ ), the linear segment is the unique shortest path between two endpoints, and therefore the unique 1-spanner of $T$ is the trivial complete graph on $T$. On the other hand, if the unit ball of the norm is a polygon (in particular, for $l_{1}$ and $l_{\infty}$ ), the points are connected by several shortest paths, therefore the problem of finding the sparsest 1-spanner becomes non trivial. In this connection, minimum Manhattan networks are precisely the optimal 1-spanners for the $l_{1}\left(\right.$ or $\left.l_{\infty}\right)$ plane. Sparse geometric spanners have applications in VLSI circuit design, network design, distributed algorithms and other areas, see for example the survey of [5]. Finally, Lam, Alexandersson, and Pachter [7] suggested to apply minimum Manhattan networks to design efficient search spaces for pair hidden Markov model (PHMM) alignment algorithms.

\section{Properties and LP-formulation}

In this section, we present several properties of minimum Manhattan networks. First, we define our notation. Denote by $[p, q]$ the linear segment having $p$ and $q$ as end-points. The set of all points of $\mathbb{R}^{2}$ lying on $l_{1}$-paths between $p$ and $q$ constitute the smallest axis-parallel closed rectangle $R(p, q)$ containing the points $p, q$. For two terminals $t_{i}, t_{j} \in T$, let $R_{i, j}:=R\left(t_{i}, t_{j}\right)$. (This rectangle is degenerate if $t_{i}$ and $t_{j}$ have the same $x$ - or $y$-coordinate.) We say that $R_{i, j}$ is an empty rectangle if $R_{i, j} \cap T=\left\{t_{i}, t_{j}\right\}$. The complete grid is obtained by drawing in the smallest axis-parallel rectangle containing the set $T$ a horizontal segment and a vertical segment through every terminal which span the entire length and width of the rectangle. The following result can be easily proven using standard methods for establishing Hanan grid-type results.

Lemma 2.1 [4, 14] The complete grid contains at least one minimum Manhattan network on $T$.

A point $p \in \mathbb{R}^{2}$ is said to be an efficient point of $T[3,13]$ if there does not exist any other point $q \in \mathbb{R}^{2}$ such that $d\left(q, t_{i}\right) \leq d\left(p, t_{i}\right)$ for each $t_{i} \in T$ and $d\left(q, t_{j}\right)<d\left(p, t_{j}\right)$ for at least one $t_{j} \in T$. Denote the set of all efficient points by $\mathcal{P}$, called the Pareto envelope of $T$. An optimal $O(n \log n)$ time algorithm to compute the Pareto envelope of $n$ points in the $l_{1}$-plane 


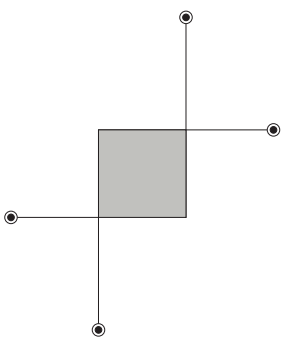

(a)

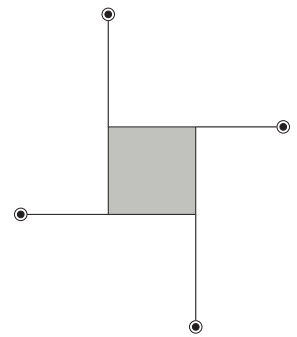

(b)

Figure 2: Pareto envelope of four points

is presented in [3]. Its correctness uses the following characterization of $\mathcal{P}$ presented in [3]: $\mathcal{P}=\bigcap_{i=1}^{n} \bigcup_{j=1}^{n} R\left(t_{i}, t_{j}\right)$. For other properties of $\mathcal{P}$ and an $O\left(n^{2}\right)$ time algorithm see also [13]. In particular, it is known that $\mathcal{P}$ is ortho-convex, i.e. the intersection of $\mathcal{P}$ with any vertical or horizontal line is convex, and that every two points of $\mathcal{P}$ can be joined in $\mathcal{P}$ by an $l_{1}$-path. $\mathcal{P}$, being ortho-convex, is a union of ortho-convex (possibly degenerate) rectilinear polygons (called blocks) glued together along vertices (they become cut points of $\mathcal{P}$ ); Fig. 2 presents two generic forms of the Pareto envelope of four points. Denote by $\Gamma=(V, E)$ the part of the complete grid contained in the Pareto envelope $\mathcal{P}$.

Lemma 2.2 The graph $\Gamma$ contains at least one minimum Manhattan network on $T$.

Proof. By Lemma 2.1, the complete grid contains a minimum Manhattan network $N$ on $T$. Denote by $\mathcal{R}(N)$ the union of all inner faces of $N$. Suppose that $N$ is selected so that to minimize the number of faces of the complete grid which belong to $\mathcal{R}(N) \backslash \mathcal{P}$. If some vertices and edges of $N$ are outside $\Gamma$, then necessarily $\mathcal{R}(N) \backslash \mathcal{P} \neq \emptyset$. Let $u_{0}$ be a vertex of $N$ located outside $\Gamma$ (and $\mathcal{P}$ ). Since $\mathcal{P}=\bigcap_{i=1}^{n} \bigcup_{j=1}^{n} R\left(t_{i}, t_{j}\right)$ and $u_{0} \notin \mathcal{P}$, there exists a terminal $t_{i}$ such that $u_{0} \notin \bigcup_{j=1}^{n} R\left(t_{i}, t_{j}\right)$. Suppose without loss of generality that $u_{0}^{x} \leq t_{i}^{x}$ and $t_{i}^{y} \leq u_{0}^{y}$. Then $u_{0} \notin \bigcup_{j=1}^{n} R\left(t_{i}, t_{j}\right)$ implies that the closed quadrant $\mathcal{Q}_{0}=\left\{p: p^{x} \leq u_{0}^{x}\right.$ and $\left.u_{0}^{y} \leq p^{y}\right\}$ does not contain any terminal of $T$. Therefore $\mathcal{Q}_{0} \bigcap\left(\bigcup_{j=1}^{n} R\left(t_{i}, t_{j}\right)\right)=\emptyset$, yielding $\mathcal{Q}_{0} \cap \mathcal{P}=\emptyset$.

Let $u$ be the highest vertex of $N \backslash \Gamma$ belonging to $\mathcal{Q}_{0}$ (if there are several such vertices, then we break ties by taking the leftmost one). The closed quadrant $\mathcal{Q}=\left\{p: p^{x} \leq u^{x}\right.$ and $\left.u^{y} \leq p^{y}\right\}$ does not contain terminals or Pareto points because $\mathcal{Q} \subseteq \mathcal{Q}_{0}$. Since $u$ is a vertex of the complete grid, the horizontal line $l_{h}$ passing via $u$ contains some terminal $t^{\prime}$ (necessarily located on the right of $u$ ). Analogously, the vertical line $l_{v}$ passing via $u$ contains some terminal $t^{\prime \prime}$ (necessarily located below $u$ ). Since $\mathcal{Q} \cap \Gamma=\emptyset$, from the choice of $u$ we infer that $\mathcal{Q} \cap N=\{u\}$. Therefore the vertex $u$ has exactly two neighbors $v$ and $w$ in $N: v$ is to the right of $u$ and $w$ is below $u$ (see Fig. 3(a)). Since $u \in N \backslash \Gamma$, the edges $[u, v]$ and $[u, w]$ do not belong to $\Gamma$ (however, one or both $v, w$ maybe vertices of this graph). Pick the point $z=\left(v^{x}, w^{y}\right)$. Since $v$ and $w$ are vertices of the complete grid, $z$ is also a vertex of this grid. Denote by $N^{\prime}$ the rectilinear network (of length at most $l(N)$ ) which is obtained from $N$ by removing the edges $u v$ and $u w$ and adding the vertical edge $v z$ and the horizontal edge $w z$. We claim that $N^{\prime}$ is a Manhattan 


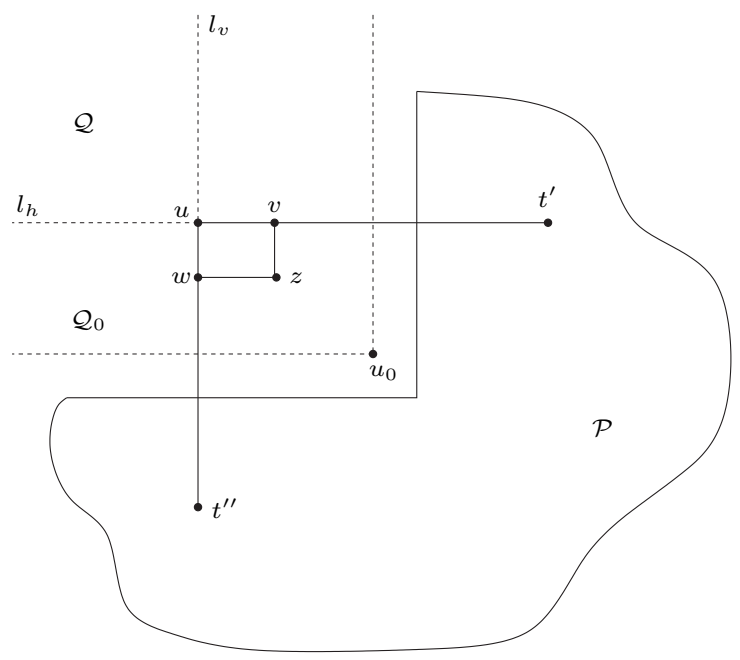

(a)

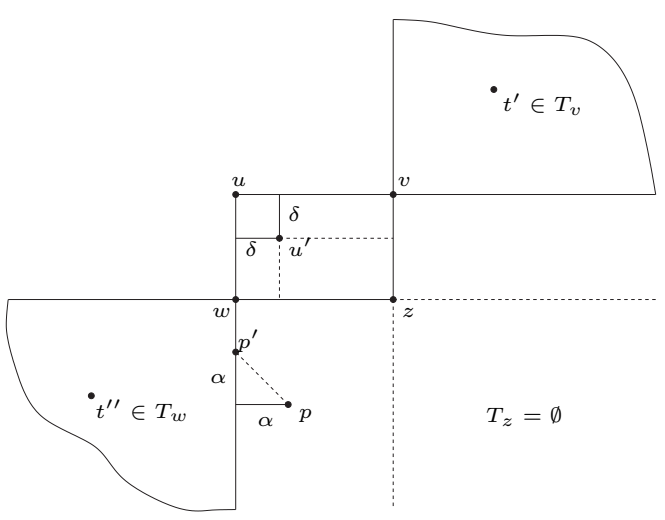

(b)

Figure 3: For the proof of Lemmata 2.2 and 2.3

network on $T$. Indeed, since all points of $T$ are located inside or on the boundary of $\mathcal{P}$, the removed vertex $u$ is not a terminal. Additionally, since the degree of $u$ in $N$ is two, any $l_{1}$-path $L$ connecting two terminals and passing via $u$ uses both edges $u v$ and $u w$. Therefore the path $L^{\prime}$ obtained from $L$ by replacing the edges $v u$ and $u w$ of $N$ by the edges $v z$ and $z w$ of $N^{\prime}$ is an $l_{1}$-path between the same pair of terminals. All this shows that $N^{\prime}$ is also a minimum Manhattan network contained in the complete grid. Since the rectangle $u v z w$ is a face of the complete grid contained in $\mathcal{R}(N) \backslash \mathcal{P}$ but not in $\mathcal{R}\left(N^{\prime}\right)$, we get a contradiction with the choice of $N$.

By this result, in order to solve the MMN problem on $T$ it suffices to complete the set of terminals by adding to $T$ the cut points of $\mathcal{P}$ and to solve a MMN problem on each block of $\mathcal{P}$ with respect to the new and old terminals located inside or on its boundary. Due to this decomposition of the MMN problem into smaller subproblems, further in this paper we can assume without loss of generality that $\mathcal{P}$ consists of a single block with at least 3 terminals; denote by $\partial \mathcal{P}$ the boundary of this ortho-convex rectilinear polygon.

Lemma 2.3 Let $\mathcal{P}$ be a block. Then (i) every convex vertex of $\mathcal{P}$ is a terminal of $T$ and (ii) the subpath $P\left(t_{i}, t_{j}\right)$ of $\partial \mathcal{P}$ between two consecutive convex vertices $t_{i}, t_{j}$ of $\partial \mathcal{P}$ is the unique $l_{1}$-path connecting the terminals $t_{i}, t_{j}$ inside $\mathcal{P}$.

Proof. In order to prove (i), suppose by way of contradiction that $u$ is a convex vertex of $\mathcal{P}$ but not a terminal. Then $u$ has exactly two neighbors $v, w$ in $\Gamma$. Suppose without loss of generality that $v$ is to the right of $u$ and $w$ is below $u$. Let $z=\left(v^{x}, w^{y}\right)$. Since $u$ is a convex vertex of $\mathcal{P}$, the face $u v z w$ of the complete grid belongs to $\mathcal{P}$. Pick $0<\delta \leq \min \{d(u, v), d(u, w)\}$ and let $u^{\prime}=\left(u^{x}-\delta, u^{y}-\delta\right)$. Now, we divide $T$ into three subsets: $T_{z}$ consists of all terminals 
$t \in T$ which can be connected to $u$ using an $l_{1}$-path passing via $z, T_{v}$ consists of all terminals $t \in T \backslash T_{z}$ such that any $l_{1}$-path connecting $t$ to $u$ passes via $v$, and, finally, $T_{w}$ consists of all terminals $t \in T \backslash T_{z}$ such that any $l_{1}$-path connecting $t$ to $u$ passes via $w$. Notice that the point $u^{\prime}$ has the same distance as $u$ to any terminal from $T_{v} \cup T_{w}$ and $u^{\prime}$ is closer than $u$ from any terminal of $T_{z}$. Since $u^{\prime}$ cannot dominate $u$ because $u \in \mathcal{P}$, we conclude that $T_{z}=\emptyset$. Therefore any terminal $t$ of $T$ either is located above and to the right of $v$ (i.e., $t \in T_{v}$ ) or is located below and to the left of $w$ (i.e., $t \in T_{w}$ ). In this case, it can be easily shown that every point $p$ lying in the open quadrant $\left\{\left(p^{x}, p^{y}\right): p^{x}>w^{x}, p^{y}<w^{y}\right\}$ is dominated by the point $p^{\prime}=\left(p^{x}-\alpha, p^{y}+\alpha\right)$ where $\alpha=\min \left\{p^{x}-w^{x}, w^{y}-p^{y}\right\}$ (see Fig. 3(b)). Analogously, every point lying in the three open quadrants

$$
\left\{\left(q^{x}, q^{y}\right): q^{x}>v^{x}, q^{y}<v^{y} \text { or } q^{x}<w^{x}, q^{y}>w^{y} \text { or } q^{x}<v^{x}, q^{y}>v^{y}\right\}
$$

is dominated. Therefore $\mathcal{P}$ is decomposable, namely it consists of a rectangular block $u v z w$, one or several blocks located above and to the right of $v$, and one or several blocks located below and to the left of $w$. This contradicts the assumption that $\mathcal{P}$ is a block, thus establishing the property (i).

To show (ii), note that the path $P\left(t_{i}, t_{j}\right)$ between two consecutive convex vertices of $\mathcal{P}$ is either a single vertical or horizontal segment or it consists of two segments, one vertical and another horizontal. In the first case, $P\left(t_{i}, t_{j}\right)=R\left(t_{i}, t_{j}\right)$ and we are done. In the second case, the segments constituting $P\left(t_{i}, t_{j}\right)$ are incident sides of the rectangle $R\left(t_{i}, t_{j}\right)$. Moreover, $\mathcal{P} \cap R\left(t_{i}, t_{j}\right)=P\left(t_{i}, t_{j}\right)$, otherwise between $t_{i}$ and $t_{j}$ will be located yet another convex vertex of $\mathcal{P}$, which is impossible. Thus $P\left(t_{i}, t_{j}\right)$ is the unique $l_{1}$-path between $t_{i}$ and $t_{j}$ in the Pareto envelope $\mathcal{P}$.

Since $\partial \mathcal{P}$ is covered by the $l_{1}$-paths $P\left(t_{i}, t_{j}\right)$ between consecutive convex vertices $t_{i}, t_{j}$ of $\partial \mathcal{P}$, from Lemmata 2.2 and 2.3 we obtain the following result:

Lemma 2.4 The edges of $\partial \mathcal{P}$ belong to any minimum Manhattan network located inside $\Gamma$.

Two edges of $\Gamma=(V, E)$ are called twins if they are opposite edges of a rectangular face of the grid $\Gamma$. Two edges $e, f$ of $\Gamma$ are called parallel if there exists a sequence $e=$ $e_{1}, e_{2}, \ldots, e_{m+1}=f$ of edges such that for $i=1, \ldots, m$ the edges $e_{i}, e_{i+1}$ are twins. By definition, any edge $e$ is parallel to itself and all edges parallel to $e$ have the same length. Notice also that from the ortho-convexity of $\mathcal{P}$ follows that exactly two edges parallel to a given edge $e$ belong to $\partial \mathcal{P}$.

We continue with the notion of a generating set introduced in [6] and used in approximation algorithms from $[1,8]$. A generating set is a subset $F$ of pairs of terminals (or, more compactly, of their indices) with the property that a rectilinear network containing $l_{1}$-paths for all pairs in $F$ is a Manhattan network on $T$. For example, $F_{\varnothing}$ consisting of all pairs $i, j$ with $R_{i, j}$ empty is a generating set [6]. In the next section, we will describe a generating set which is a subset of $F_{\varnothing}$. 
To give an LP-formulation of the minimum Manhattan network problem, let $\vec{F}$ be an arbitrary generating set whose pairs are ordered in an arbitrary way; for each ordered pair $(i, j) \in \vec{F}$, let $\Gamma_{i, j}:=\Gamma \cap R\left(t_{i}, t_{j}\right)$ and set $\Gamma_{i, j}=\left(V_{i, j}, E_{i, j}\right)$. We formulate the MMN problem as a cut covering problem using an exponential number of constraints, which we further convert into an equivalent formulation that employs only a polynomial number of variables and constraints. In both formulations, $l_{e}$ will denote the length of an edge $e$ of the network $\Gamma=(V, E)$ and $x_{e}$ will be a $0-1$ decision variable associated with $e$. A subset of edges $C$ of $E_{i, j}$ is called a $\left(t_{i}, t_{j}\right)$-cut if every $l_{1}$-path between $t_{i}$ and $t_{j}$ in $\Gamma_{i, j}$ shares an edge with $C$. Let $\mathcal{C}_{i, j}$ denote the collection of all $\left(t_{i}, t_{j}\right)$-cuts and set $\mathcal{C}:=\bigcup_{(i, j) \in \vec{F}} \mathcal{C}_{i, j}$. Then the minimum Manhattan networks can be viewed as the optimal solutions of the following integer linear program (the dual of the relaxation of this program is a packing problem of the cuts from $\mathcal{C}$ ):

$$
\begin{array}{lll}
\operatorname{minimize} & \sum_{e \in E} l_{e} x_{e} & \\
\text { subject to } & \sum_{e \in C} x_{e} \geq 1, \quad C \in \mathcal{C} \\
& x_{e} \in\{0,1\}, \quad e \in E
\end{array}
$$

Indeed, every Manhattan network is a feasible solution of (1). Conversely, let $x_{e}, e \in E$, be a feasible solution for (1). Considering $x_{e}$ 's as capacities of the edges $e$ of $\Gamma$, and applying the covering constraints and the Ford-Fulkerson's theorem to each network $\Gamma_{i, j},(i, j) \in \vec{F}$, oriented as described below, we conclude the existence in $\Gamma_{i, j}$ of an integer $\left(t_{i}, t_{j}\right)$-flow of value 1 , i.e., of an $l_{1}$-path between $t_{i}$ and $t_{j}$. As a consequence, we obtain a Manhattan network of the same cost. This observation leads to the second integer programming formulation for the MMN problem (but this time, having a polynomial size). For each pair $(i, j) \in \vec{F}$ and each edge $e \in E_{i, j}$ introduce a (flow) variable $f_{e}^{i, j}$. Orient the edges of $\Gamma_{i, j}$ so that the oriented paths connecting $t_{i}$ and $t_{j}$ are exactly the $l_{1}$-paths between those terminals (notice that this orientation is not overall consistent in the sense that the same edge may be oriented in different ways in different grids to which it belongs). For a vertex $v \in V_{i, j} \backslash\left\{t_{i}, t_{j}\right\}$ denote by $\Gamma_{i, j}^{+}(v)$ the oriented edges of $\Gamma_{i, j}$ entering $v$ and by $\Gamma_{i, j}^{-}(v)$ the oriented edges of $\Gamma_{i, j}$ out of $v$. We obtain the following integer program:

$$
\begin{aligned}
& \text { minimize } \quad \sum_{e \in E} l_{e} x_{e} \\
& \text { subject to } \quad \sum_{e \in \Gamma_{i, j}^{+}(v)} f_{e}^{i, j}=\sum_{e \in \Gamma_{i, j}^{-}(v)} f_{e}^{i, j},(i, j) \in \vec{F}, v \in V_{i, j} \backslash\left\{t_{i}, t_{j}\right\} \\
& \sum_{e \in \Gamma_{i, j}^{-}\left(t_{i}\right)} f_{e}^{i, j}=1, \quad(i, j) \in \vec{F} \\
& 0 \leq f_{e}^{i, j} \leq x_{e}, \quad(i, j) \in \vec{F}, \forall e \in E_{i, j} \\
& x_{e} \in\{0,1\}, \quad e \in E
\end{aligned}
$$


Denote by $\left(1^{\prime}\right)$ and $\left(2^{\prime}\right)$ the LP-relaxation of (1) and (2) obtained by replacing the boolean constrains $x_{e} \in\{0,1\}$ by the linear constraints $x_{e} \geq 0$. (The constraint $x_{e} \leq 1$ was omitted because in any optimal solution for each $x_{e}$ at least one constraint $f_{e} \leq x_{e}$ is tight and $f_{e}^{i, j} \leq 1$ by first and second equalities.) Since $\left(2^{\prime}\right)$ contains a polynomial number of variables and inequalities, it can be solved in strongly polynomial time using the algorithm of Tardos [10]. The $x$-part of any optimal solution $(\mathbf{x}, \mathbf{f})$ of $\left(2^{\prime}\right)$ is an optimal solution of $\left(1^{\prime}\right)$. It can be viewed as a "fractional Manhattan network" in the following sense. In the network $\Gamma_{i, j}$ endowed with capacities $x_{e}, e \in E_{i, j}$, for each pair $\{i, j\}$, there exists one or several $l_{1}$-paths carrying flow of total value $\geq 1$. If the optimal solution $\mathbf{x}$ is integral, i.e. $x_{e} \in\{0,1\}, e \in E$, then every such flow uses a unique $l_{1}$-path and therefore $\mathbf{x}$ is the characteristic vector of an optimal Manhattan network. Unfortunately, this is not always the case, morever there exist instances of the MMN problem for which the cost of an optimal (fractional) solution of $\left(1^{\prime}\right)$ or $\left(2^{\prime}\right)$ is smaller than the cost of an optimal (integer) solution of (1) or (2). Fig. 4 shows such an example $\left(x_{e}=1\right.$ for heavy edges and $x_{e}=\frac{1}{2}$ for dashed edges). Notice also that by Lemma 2.4 in any feasible solution of $\left(1^{\prime}\right)$ and $\left(2^{\prime}\right)$ for any edge $e \in \partial \mathcal{P}$ it holds that $x_{e}=1$.

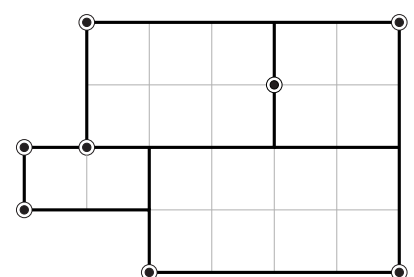

Integer optimum $=28$

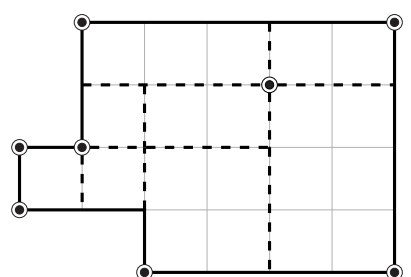

Fractional optimum $=27.5$

Figure 4: Integrality gap

\section{Strips and staircases}

A degenerate empty rectangle $R_{i, j}$ is called a degenerate vertical or horizontal strip. A nondegenerate empty rectangle $R_{i, j}$ is called a vertical strip if there exists no terminal in $T$ with $x$-coordinate strictly between the $x$-coordinates of $t_{i}$ and $t_{j}$ and the intersection of $R_{i, j}$ with degenerate vertical strips is either empty or one of the points $t_{i}$ or $t_{j}$. The first condition means that the $x$-coordinates of $t_{i}$ and $t_{j}$ are consecutive entries of the sorted list of all distinct $x$ coordinates of the terminals. The second condition means that either $t_{i}$ is the highest terminal among all terminals having the same $x$-coordinate as $t_{i}$ and $t_{j}$ is the lowest terminal among all terminals having the same $x$-coordinates as $t_{j}$, or, vice-versa, $t_{i}$ is the lowest terminal and $t_{j}$ is the highest terminal among respective terminals; see Fig. 5 for an illustration. The second condition ensures that each subset of pairwise parallel horizontal edges may intersect at most 


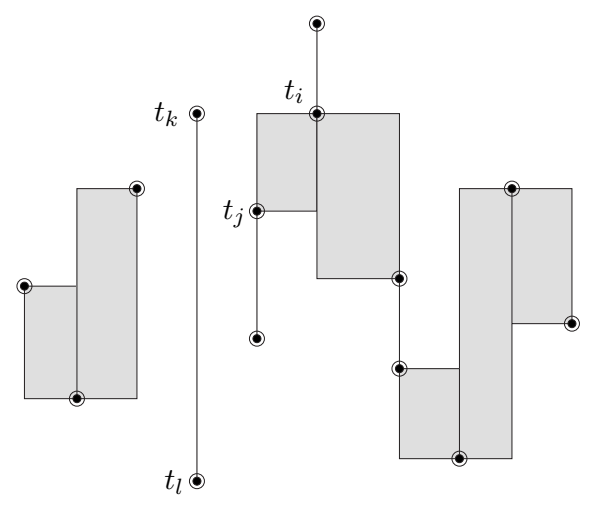

Figure 5: $R_{i, j}$ is a vertical strip, while neither $R_{j, k}$ nor $R_{j, l}$ are

one vertical strip. Analogously, a non-degenerate empty rectangle $R_{i, j}$ is called a horizontal strip if there exists no terminal in $T$ with $y$-coordinate between the $y$-coordinates of $t_{i}$ and $t_{j}$ and the intersection of horizontal sides of $R_{i, j}$ with degenerate horizontal strips is either empty or one of the points $t_{i}$ or $t_{j}$. The sides of a vertical (resp., horizontal) strip $R_{i, j}$ are the vertical (resp., horizontal) sides of $R_{i, j}$. Notice that two points $t_{i}, t_{j}$ may define both a horizontal and a vertical strip. We say that the strips $R_{i, i^{\prime}}$ and $R_{j, j^{\prime}}$ (degenerate or not) form a crossing configuration if they intersect and the Pareto envelope of the points $t_{i}, t_{i^{\prime}}, t_{j}, t_{j^{\prime}}$ is of type (a); see Fig. 2. The importance of such configurations resides in the following property whose proof is straightforward:

Lemma 3.1 If the rectangles $R_{i, i^{\prime}}$ and $R_{j, j^{\prime}}$ form a crossing configuration as in Fig. 6, then from the $l_{1}$-paths between $t_{i}$ and $t_{i^{\prime}}$ and between $t_{j}$ and $t_{j^{\prime}}$ one can derive an $l_{1}$-path connecting $t_{i}$ and $t_{j^{\prime}}$ and an $l_{1}$-path connecting $t_{i^{\prime}}$ and $t_{j}$.

For a crossing configuration $R_{i, i^{\prime}}, R_{j, j^{\prime}}$, denote by $o$ and $o^{\prime}$ the cut points of the rectangular block of the Pareto envelope of $t_{i}, t_{i^{\prime}}, t_{j}, t_{j^{\prime}}$, and assume that the four tips of this envelope connect $o$ with $t_{i}, t_{j}$ and $o^{\prime}$ with $t_{i^{\prime}}, t_{j^{\prime}}$. Additionally, suppose without loss of generality, that $t_{i}$ and $t_{j}$ belong to the first quadrant $\mathcal{Q}_{1}$ with respect to the origin $o$ (the remaining quadrants are labelled $\mathcal{Q}_{2}, \mathcal{Q}_{3}$, and $\mathcal{Q}_{4}$ in counterclockwise order around $o$ ). Then $t_{i^{\prime}}$ and $t_{j^{\prime}}$ belong to the third quadrant with respect to the origin $o^{\prime}$. Denote by $T_{i, j}$ the set of all terminals $t_{k} \in\left(T \backslash\left\{t_{i}, t_{j}\right\}\right) \cap \mathcal{Q}_{1}$ such that (i) $R\left(t_{k}, o\right) \cap T=\left\{t_{k}\right\}$ and (ii) the region $\left\{q \in \mathcal{Q}_{2}: q^{y} \leq\right.$ $\left.t_{k}^{y}\right\} \cup\left\{q \in \mathcal{Q}_{4}: q^{x} \leq t_{k}^{x}\right\}$ does not contain any terminal of $T$. If $T_{i, j}$ is nonempty, then all its terminals belong to the rectangle $R_{i, j}$, more precisely, they are all located on a common shortest rectilinear path between $t_{i}$ and $t_{j}$. Denote by $\mathcal{S}_{i, j \mid i^{\prime}, j^{\prime}}$ the non-degenerate block of the Pareto envelope of the set $T_{i, j} \cup\left\{o, t_{i}, t_{j}\right\}$ and call this rectilinear polygon a staircase; see Fig. 6 for an illustration. The point $o$ is called the origin of this staircase. Analogously one can define the set $T_{i^{\prime}, j^{\prime}}$ and the staircase $\mathcal{S}_{i^{\prime}, j^{\prime} \mid i, j}$ with origin $o^{\prime}$. Two other types of staircases will be defined if $t_{i}, t_{j}$ belong to the second quadrant with respect to $o$ and $t_{i^{\prime}}, t_{j^{\prime}}$ belong to the fourth quadrant with respect to $o^{\prime}$. In order to simplify the presentation, further we will 


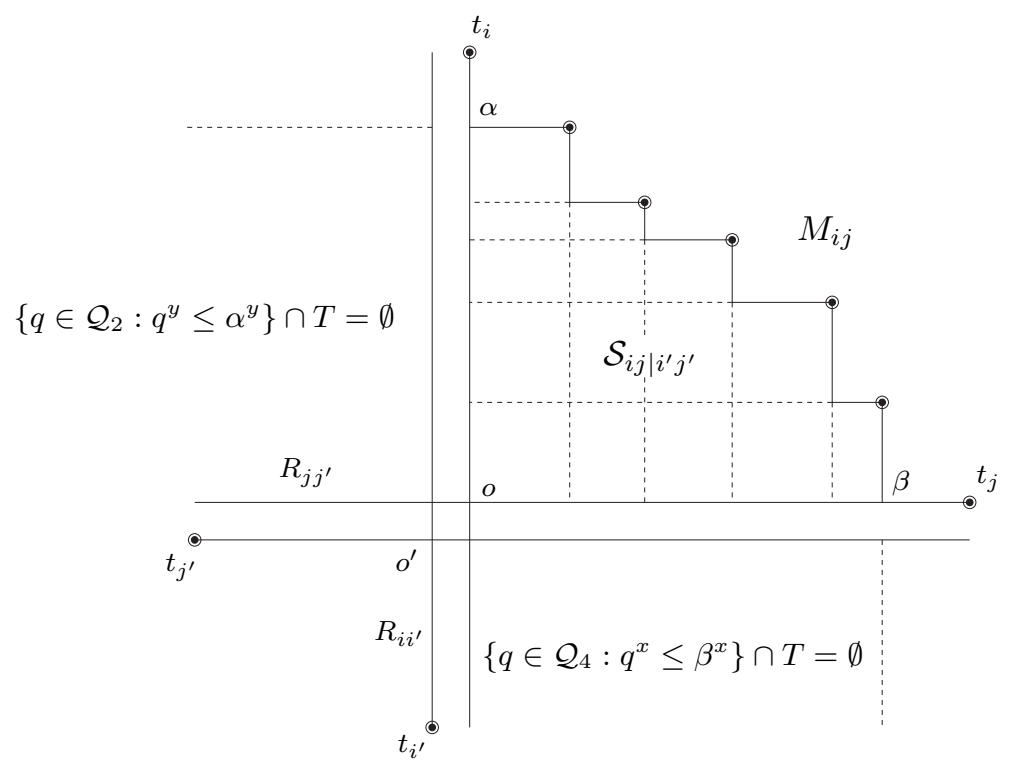

Figure 6: Staircase $\mathcal{S}_{i, j \mid i^{\prime}, j^{\prime}}$

prove all results under the assumption that the staircase is located in the first quadrant. By symmetry, all these results also hold for other types of staircases. (Notice that our staircases are different from the staircase polygons occurring in the algorithms from [4].)

Let $\alpha$ be the leftmost highest point of the staircase $\mathcal{S}_{i, j \mid i^{\prime}, j^{\prime}}$ and let $\beta$ be the rightmost lowest point of this staircase. Denote by $M_{i, j}$ the monotone boundary path of $\mathcal{S}_{i, j \mid i^{\prime}, j^{\prime}}$ between $\alpha$ and $\beta$ and passing via the terminals of $T_{i, j}$. By definition, $\mathcal{S}_{i, j \mid i^{\prime}, j^{\prime}} \cap T=T_{i, j}$. By the choice of $T_{i, j}$, there are no terminals of $T$ located in the regions $\left\{q \in \mathcal{Q}_{2}: q^{y} \leq \alpha^{y}\right\}$ and $\left\{q \in \mathcal{Q}_{4}: q^{x} \leq \beta^{x}\right\}$. In particular, no strip traverses a staircase. From the definition of a staircase, it immediately follows that two staircases either are disjoint or their intersection is a subset of vertices of both of them (all these vertices are terminals); in particular, every edge of the grid $\Gamma$ belongs to at most one staircase.

Let $F^{\prime}$ be the set of all pairs $\{i, j\}$ such that $R_{i, j}$ is a strip. Let $F^{\prime \prime}$ be the set of all pairs $\left\{i^{\prime}, k\right\}$ such that there exists a staircase $\mathcal{S}_{i, j \mid i^{\prime}, j^{\prime}}$ such that $t_{k}$ belongs to the set $T_{i, j}$. From the definition of strips and staircases immediately follows that $F^{\prime} \cup F^{\prime \prime} \subseteq F_{\varnothing}$.

Lemma $3.2 F:=F^{\prime} \cup F^{\prime \prime}$ is a generating set.

Proof. Let $N$ be a rectilinear network containing $l_{1}$-paths for all pairs in $F$. To prove that $N$ is a Manhattan network on $T$, it suffices to establish that for an arbitrary pair $\left\{k, k^{\prime}\right\} \in F_{\varnothing} \backslash F$, the terminals $t_{k}$ and $t_{k^{\prime}}$ are joined in $N$ by an $l_{1}$-path. Assume without loss of generality that $t_{k^{\prime}}^{x} \leq t_{k}^{x}$ and $t_{k^{\prime}}^{y} \leq t_{k}^{y}$. The vertical and horizontal lines through the points $t_{k}$ and $t_{k^{\prime}}$ partition the plane into the rectangle $R_{k, k^{\prime}}$, four open quadrants and four closed unbounded half-bands labelled counterclockwise $\mathcal{B}_{1}, \mathcal{B}_{2}, \mathcal{B}_{3}$, and $\mathcal{B}_{4}$. Consider the leftmost terminal $t_{i_{1}}$ of $\mathcal{B}_{1}$, breaking ties by minimizing the $y$-coordinate (this terminal exists because $t_{k} \in \mathcal{B}_{1}$ ). Now, 


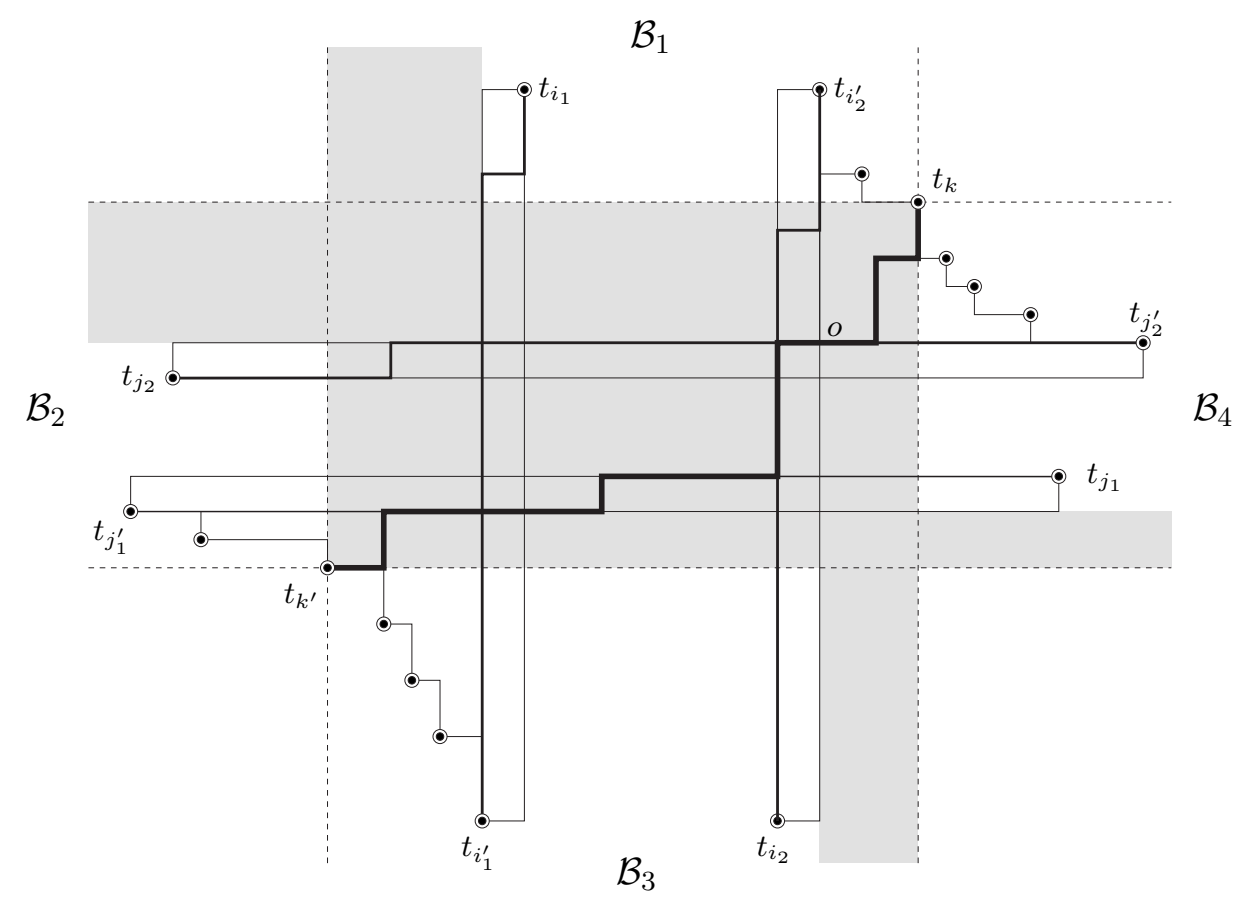

Figure 7: For the proof of Lemma 3.2

consider the rightmost terminal $t_{i_{1}^{\prime}}$ of $\mathcal{B}_{3}$ such that $t_{i_{1}^{\prime}}^{x} \leq t_{i_{1}}^{x}$, breaking ties by maximizing the $y$-coordinate (again this terminal exists because $t_{k^{\prime}} \in \mathcal{B}_{3}$ and $t_{k^{\prime}}^{x} \leq t_{i_{1}}^{x}$ ). By the choice of $t_{i_{1}}$ and $t_{i_{1}^{\prime}}$, the rectangle $R_{i_{1}, i_{1}^{\prime}}$ is the leftmost vertical strip crossing the rectangle $R_{k, k^{\prime}}$. Analogously, by letting $t_{i_{2}}, t_{j_{1}}$, and $t_{j_{2}}$ be the rightmost terminal of $\mathcal{B}_{3}$, the highest terminal of $\mathcal{B}_{2}$, and the lowest terminal of $\mathcal{B}_{4}$, respectively (minimizing the distance to $R_{k, k^{\prime}}$ in case of ties), we obtain the leftmost vertical strip $R_{i_{2}, i_{2}^{\prime}}$, the lowest horizontal strip $R_{j_{1}, j_{1}^{\prime}}$, and the highest horizontal strip $R_{j_{2}, j_{2}^{\prime}}$ crossing the rectangle $R_{k, k^{\prime}}$. Notice that the strips $R_{j_{2}, j_{2}^{\prime}}$ and $R_{i_{2}, i_{2}^{\prime}}$ as well as the strips $R_{j_{1}, j_{1}^{\prime}}$ and $R_{i_{1}, i_{1}^{\prime}}$ constitute crossing configurations.

Now, we will prove that $N$ contains an $l_{1}$-path between $t_{k}$ and $t_{i_{2}}$ and an $l_{1}$-path between $t_{k^{\prime}}$ and $t_{j_{1}}$. We distinguish three cases. If $t_{k}=t_{i_{2}^{\prime}}$, then $R_{i_{2}, k}=R_{i_{2}, i_{2}^{\prime}}$ is a strip and thus $\left\{k, i_{2}\right\} \in F$. If $t_{k}=t_{j_{2}^{\prime}}$, then the strips $R_{j_{2}, k}$ and $R_{i_{2}, i_{2}^{\prime}}$ form a crossing configuration. By Lemma 3.1, from the $l_{1}$-paths of $N$ between $t_{j_{2}}$ and $t_{k}$ and between $t_{i_{2}}$ and $t_{i_{2}^{\prime}}$, we can derive an $l_{1}$-path between $t_{k}$ and $t_{i_{2}}$. Finally, if $t_{k} \notin\left\{t_{i_{2}^{\prime}}, t_{j_{2}^{\prime}}\right\}$, we assert that the crossing configuration $R_{i_{2}, i_{2}^{\prime}}$ and $R_{j_{2}, j_{2}^{\prime}}$ defines a staircase $\mathcal{S}_{i_{2}^{\prime} j_{2}^{\prime} \mid i_{2} j_{2}}$ such that $t_{k}$ belongs to $T_{i_{2}^{\prime}, j_{2}^{\prime}}$. Indeed, let $o$ be the highest leftmost intersection point of the strips $R_{i_{2}, i_{2}^{\prime}}$ and $R_{j_{2}, j_{2}^{\prime}}$ (see Fig. 7). Since $R\left(t_{k}, o\right.$ ) is contained in the empty rectangle $R\left(t_{k}, t_{k^{\prime}}\right)$, we conclude that $R\left(t_{k}, o\right) \cap T=\left\{t_{k}\right\}$. Moreover, by the choice of $t_{i_{2}}$ and $t_{j_{2}}$, the unbounded half-bands $\left\{q \in \mathcal{B}_{3}: q^{x} \geq t_{i_{2}}^{\prime}\right\}$ and $\left\{q \in \mathcal{B}_{2}: q^{y} \geq t_{j_{2}^{\prime}}\right\}$ do not contain terminals (in Fig. 7 , the shaded region does not contain terminals), thus establishing our assertion. This implies that $t_{k} \in T_{i_{2}^{\prime}, j_{2}^{\prime}}$, whence $\left\{k, i_{2}\right\} \in F$. Therefore, in all three cases the terminals $t_{k}$ and $t_{i_{2}}$ are connected in $N$ by an $l_{1}$-path. Using a similar analysis, one can show that $t_{k^{\prime}}$ and $t_{j_{1}}$ are also connected in $N$ by an $l_{1}$-path. By construction, the 
rectangles $R_{k, i_{2}}$ and $R_{k^{\prime}, j_{1}}$ form a crossing configuration and thus, by Lemma 3.1, there is an $l_{1}$-path of $N$ between the terminals $t_{k}$ and $t_{k^{\prime}}$, concluding the proof.

\section{The rounding algorithm}

Let $(\mathbf{x}, \mathbf{f})=\left(\left(x_{e}\right)_{e \in E},\left(f_{e}^{i, j}\right)_{e \in E,(i, j) \in F}\right)$ be an optimal solution of the linear program $\left(2^{\prime}\right)$ (in general, this solution is not half-integral). The algorithm rounds up the solution $(\mathbf{x}, \mathbf{f})$ in three phases. In Phase 0, we insert all edges of $\partial \mathcal{P}$ in the integer solution. In Phase 1, the rounding is performed inside every strip $R_{i, i^{\prime}}$, in order to ensure the existence of an $l_{1}$-path $P_{i, i^{\prime}}$ between the terminals $t_{i}$ and $t_{i^{\prime}}$. In Phase 2, an iterative rounding procedure is applied to each staircase.

Let $R_{i, i^{\prime}}$ be a strip. If $R_{i, i^{\prime}}$ is degenerate, then $\left[t_{i}, t_{i^{\prime}}\right]$ is the unique $l_{1}$-path between $t_{i}$ and $t_{i^{\prime}}$, yielding $x_{e}=f_{e}^{i, i^{\prime}}=1$ for any edge $e \in\left[t_{i}, t_{i^{\prime}}\right.$. If $R_{i, i^{\prime}}$ is not degenerate, then any $l_{1}$-path in $\Gamma$ between $t_{i}$ and $t_{i^{\prime}}$ has a simple form: it goes along the side of $R_{i, i^{\prime}}$ containing $t_{i}$, then it makes a turn by following an edge of $\Gamma$ traversing $R_{i, i^{\prime}}$ (called further a switch of $R_{i, i^{\prime}}$ ), and continues its way on the side containing $t_{i^{\prime}}$ until it reaches $t_{i^{\prime}}$. It may happen that several such $l_{1}$-paths have been used by the fractional flow $f^{i, i^{\prime}}$ between $t_{i}$ and $t_{i^{\prime}}$. However, since any pair $e, e^{\prime}$ of twins on opposite sides of the strip $R_{i, i^{\prime}}$ constitute a cut separating the terminals $t_{i}$ and $t_{i^{\prime}}$, the value of the $f^{i, i^{\prime}}$-flow traversing this cut is at least 1 , yielding $x_{e}+x_{e^{\prime}} \geq f_{e}^{i, i^{\prime}}+f_{e^{\prime}}^{i, i^{\prime}} \geq 1$, and therefore $\max \left\{x_{e}, x_{e^{\prime}}\right\} \geq \frac{1}{2}$.

Let $p$ be the furthest from $t_{i}$ vertex on the side of $R_{i, i^{\prime}}$ containing $t_{i}$ such that $x_{e} \geq \frac{1}{2}$ for every edge $e$ of the segment $\left[t_{i}, p\right]$. Let $p p^{\prime}$ be the edge of $\Gamma$ incident to $p$ that traverses the strip $R_{i, i^{\prime}}$. By the choice of $p$, the $\left(t_{i}, t_{i^{\prime}}\right)$-flow carried by the $l_{1}$-paths which make a turn before $p$ or at the vertex $p$ has total value $\geq \frac{1}{2}$. Since all these paths contain all edges $e$ of the segment $\left[p^{\prime}, t_{i^{\prime}}\right]$, we have $x_{e} \geq \frac{1}{2}$ for all such edges.

Phase 1 (procedure RoundStrip). For each strip $R_{i, i^{\prime}}$, if $R_{i, i^{\prime}}$ is degenerate, then take in the integer solution all edges of $\left[t_{i}, t_{i^{\prime}}\right]$, otherwise round up the edges of $\left[t_{i}, p\right]$ and $\left[p^{\prime}, t_{i^{\prime}}\right]$ and take the edge $p p^{\prime}$ as a switch of $R_{i, i^{\prime}}$; in both cases, denote by $P_{i, i^{\prime}}$ the resulting $l_{1}$-path between $t_{i}$ and $t_{i^{\prime}}$.

Let $\mathcal{S}_{i, i^{\prime} \mid j, j^{\prime}}$ be a staircase. Denote by $\phi$ the closest to $t_{i}$ common point of the $l_{1}$-paths $P_{i, i^{\prime}}$ and $P_{j, j^{\prime}}$ (this point is a corner of the rectangular face of $\Gamma$ containing the vertices $o$ and $o^{\prime}$ ). Let $P_{i, i^{\prime}}^{+}$and $P_{j, j^{\prime}}^{+}$be the sub-paths of $P_{i, i^{\prime}}$ and $P_{j, j^{\prime}}$ between $\phi$ and the terminals $t_{i}$ and $t_{j}$, respectively. Now we slightly expand the staircase $\mathcal{S}_{i, i^{\prime} \mid j, j^{\prime}}$ by considering as $\mathcal{S}_{i, i^{\prime} \mid j, j^{\prime}}$ the region bounded by the paths $P_{i, i^{\prime}}^{+}, P_{j, j^{\prime}}^{+}$, and $M_{i, j}\left(P_{i, i^{\prime}}^{+}\right.$and $P_{j, j^{\prime}}^{+}$are not included in the staircase but $M_{i, j}$ and the terminals from the set $T_{i, j}$ are). Inside $\mathcal{S}_{i, i^{\prime} \mid j, j^{\prime}}$, any flow $f^{k, i^{\prime}}$ (or $f^{k, j^{\prime}}$ ), $t_{k} \in T_{i, j}$, may be highly fractional: it may happen that several $l_{1}$-paths between $t_{k}$ and $t_{i^{\prime}}$ carry flow $f^{k, i^{\prime}}$. Any such $l_{1}$-path intersects one of the paths $P_{i, i^{\prime}}^{+}$or $P_{j, j^{\prime}}^{+}$, therefore the total $f^{k, i^{\prime}}$-flow arriving at $P_{i, i^{\prime}}^{+} \cup P_{j, j^{\prime}}^{+}$is equal to 1 . (This flow can be directed to $\phi$ via the paths $P_{i, i^{\prime}}^{+}$and $P_{j, j^{\prime}}^{+}$, and further, along the path $P_{i, i^{\prime}}$, to the terminal $t_{i^{\prime}}$ ). Therefore it remains to decide how to round up the flow $f^{k, i^{\prime}}$ inside the expanded staircase $\mathcal{S}_{i, i^{\prime} \mid j, j^{\prime}}$. For this, notice that either 


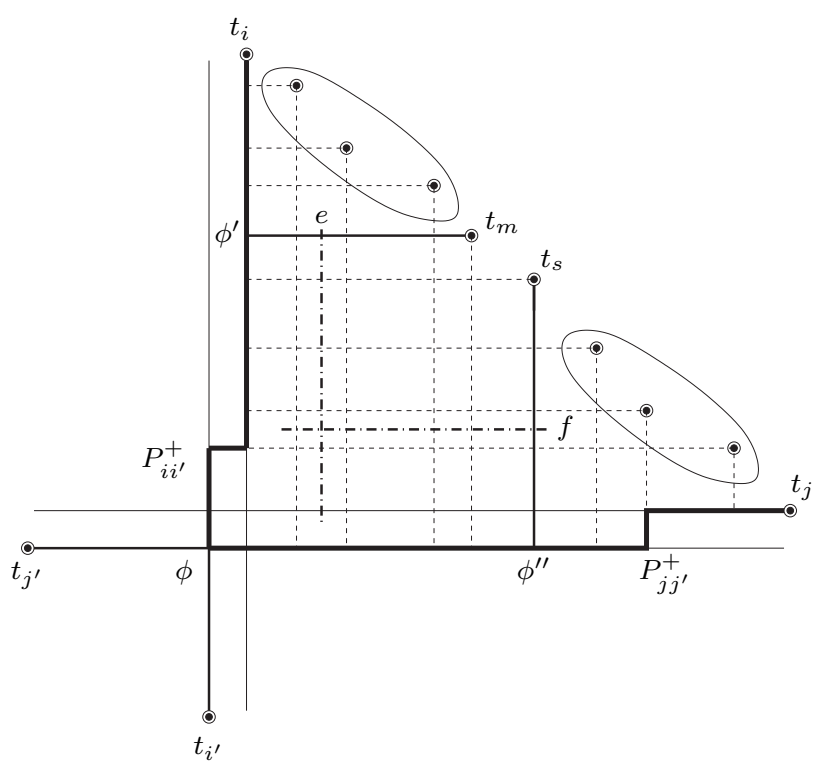

Figure 8: Procedure RoundStaircase

the total $f^{k, i^{\prime}}$-flow carried by the $l_{1}$-paths that arrive at $P_{i, i^{\prime}}^{+}$is at least $\frac{1}{2}$ or the total $f^{k, i^{\prime}}$-flow on the $l_{1}$-paths that arrive at $P_{j, j^{\prime}}^{+}$is at least $\frac{1}{2}$.

Phase 2 (procedure RoundStaircase). For a staircase $\mathcal{S}_{i, i^{\prime} \mid j, j^{\prime}}$ defined by the $l_{1}$-paths $P_{i, i^{\prime}}^{+}$and $P_{j, j^{\prime}}^{+}$and the monotone path $M_{i, j}$, find the lowest terminal $t_{m} \in T_{i, j}$ such that the $f^{m, i^{\prime}}$-flow on $l_{1}$-paths between $t_{m}$ and $t_{i^{\prime}}$ that arrive first at $P_{i, i^{\prime}}^{+}$is $\geq \frac{1}{2}$. Analogously, find the highest terminal $t_{s} \in T_{i, j}$ such that the $f^{s, i^{\prime}}$-flow on $l_{1}$-paths between $t_{s}$ and $t_{i^{\prime}}$ that arrive first at $P_{j, j^{\prime}}^{+}$is $\geq \frac{1}{2}$. Notice that at least one of the terminals $t_{m}, t_{s}$ exists and if both $t_{m}, t_{s}$ exist then they are consecutive in $M_{i j}$. Denote by $\phi^{\prime}$ the intersection of the horizontal line passing via the terminal $t_{m}$ (if it exists) with the path $P_{i, i^{\prime}}^{+}$. Analogously, let $\phi^{\prime \prime}$ denote the intersection of the vertical line passing via $t_{s}$ (if it exists) with the path $P_{j, j^{\prime}}^{+}$. If the terminal $t_{s}$ does not exist, then $t_{m}$ is the lowest terminal of $T_{i j}$ and the $f^{m i^{\prime}}$-flow on the horizontal segment $\left[t_{m}, \phi^{\prime}\right]$ is $>\frac{1}{2}$. In this case, we round up all edges of $\left[t_{m}, \phi^{\prime}\right]$. Analogously, if $t_{m}$ does not exist, then we round up the edges of the vertical segment $\left[t_{s}, \phi^{\prime \prime}\right]$. Now, suppose that both $t_{m}$ and $t_{s}$ exist. By the choice of $t_{m}$, the $f^{s, i^{\prime}}$-flow on paths which arrive at $P_{j, j^{\prime}}$ is $\geq \frac{1}{2}$. Analogously, by the choice of $t_{s}$, the $f^{m, i^{\prime}}$-flow on paths which arrive at $P_{i, i^{\prime}}$ is $\geq \frac{1}{2}$. We round up all edges of the horizontal segment $\left[t_{m}, \phi^{\prime}\right]$ and all edges of the vertical segment $\left[t_{s}, \phi^{\prime \prime}\right]$. If $T_{i, j}$ contains terminals located above the horizontal line $\left(t_{m}, \phi^{\prime}\right)$, then recursively call RoundStaircase to the expanded sub-staircase defined by $\left[t_{m}, \phi^{\prime}\right]$, the sub-path of $P_{i, i^{\prime}}^{+}$between $\phi^{\prime}$ and $t_{i}$, and the sub-path $M_{i, m}$ of the monotone path $M_{i, j}$ between $t_{m}$ and $\alpha$. Analogously, if $T_{i, j}$ contains terminals located to the right of the vertical line $\left(t_{s}, \phi^{\prime \prime}\right)$, then recursively call RoundStaircase to the expanded sub-staircase defined by $\left[t_{s}, \phi^{\prime \prime}\right]$, the sub-path of $P_{j, j^{\prime}}^{+}$comprised between $\phi^{\prime \prime}$ and $t_{j}$, and the sub-path $M_{s, j}$ of the monotone path $M_{i, j}$ between $t_{s}$ and $\beta$; see Fig. 8 for an 
illustration.

Let $E_{0}$ denote the edges of $\Gamma$ which belong to the boundary of the Pareto envelope of $T$. Let $E_{1}$ be the set of all edges picked by the procedure RoundStrip and which do not belong to $E_{0}$, and let $E_{2}$ be the set of all edges picked by the recursive procedure RoundStaircase and which do not belong to $E_{0} \cup E_{1}$. Denote by $N^{*}=\left(V^{*}, E_{0} \cup E_{1} \cup E_{2}\right)$ the resulting rectilinear network. From Lemma 3.2 and the rounding procedures presented above we infer that $N^{*}$ is a Manhattan network. Let $\mathbf{x}^{*}$ be the integer solution of (1) associated with $N^{*}$, i.e., $x_{e}^{*}=1$ if $e \in E_{0} \cup E_{1} \cup E_{2}$ and $x_{e}^{*}=0$ otherwise.

\section{Analysis}

In this section, we will show that the length of the Manhattan network $N^{*}$ is at most twice the cost of the optimal fractional solution of $\left(1^{\prime}\right)$, i.e., that

$$
\operatorname{cost}\left(\mathbf{x}^{*}\right)=\sum_{e \in E} l_{e} x_{e}^{*} \leq 2 \sum_{e \in E} l_{e} x_{e}=2 \operatorname{cost}(\mathbf{x})
$$

To establish the inequality (3), to every edge $e \in E_{1} \cup E_{2}$ we will assign a set $E_{e}$ of parallel to $e$ edges such that (i) $\sum_{e^{\prime} \in E_{e}} x_{e^{\prime}} \geq \frac{1}{2}$ and (ii) $E_{e} \cap E_{f}=\emptyset$ for any two edges $e, f \in E_{1} \cup E_{2}$ (the edges of $E_{e}$ will pay for the inclusion of the edge $e$ in $N^{*}$ ). By Lemma 2.4 the equality $x_{e}=x_{e}^{*}=1$ holds for every edge $e \in E_{0}$, thus every such edge $e$ can pay one half of $x_{e}$ for itself. The another half of $x_{e}$ can be recycled to pay an edge from $E_{1} \cup E_{2}$, namely it will be used to pay some switch.

First pick an edge $e \in E_{1}$, say $e \in P_{i, i^{\prime}}$ for a strip $R_{i, i^{\prime}}$. If $e$ belongs to a side of this strip, then $x_{e} \geq \frac{1}{2}$, and in this case we can set $E_{e}:=\{e\}$. Now, if $e$ is the switch of $R_{i, i^{\prime}}$, then $E_{e}$ consists of one of the two edges of $\partial \mathcal{P}$ parallel to $e$. From the definition of strips one concludes that no other switch can be parallel to these edges of $\partial \mathcal{P}$. Therefore each edge of $\partial P$ may appear in at most one set $E_{e}$ for a switch $e$.

Finally suppose that $e \in E_{2}$, say $e$ belongs to the expanded staircase $\mathcal{S}_{i, i^{\prime} \mid j, j^{\prime}}$. If $e$ belongs to the segment $\left[t_{m}, \phi^{\prime}\right]$, then $E_{e}$ consists of $e$ and all parallel to $e$ edges of $\mathcal{S}_{i, i^{\prime} \mid j, j^{\prime}}$ located below $e$; see Fig. 8 . Since every $l_{1}$-path between $t_{m}$ and $t_{i^{\prime}}$ intersecting the path $P_{i, i^{\prime}}^{+}$contains an edge of $E_{e}$, we infer that the value of the $f^{m, i^{\prime}}$-flow traversing the set $E_{e}$ is at least $\frac{1}{2}$, therefore $\sum_{e^{\prime} \in E_{e}} x_{e^{\prime}} \geq \frac{1}{2}$, thus establishing (i). Analogously, if $f$ is an edge of the vertical segment $\left[t_{s}, \phi^{\prime \prime}\right]$, then $E_{f}$ consists of $f$ and all parallel to $f$ edges of $\mathcal{S}_{i, i^{\prime} \mid j, j^{\prime}}$ located to the left of $f$. Obviously, $E_{e} \cap E_{f}=\emptyset$. Since $E_{e}$ and $E_{f}$ belong to the region of $\mathcal{S}_{i, i^{\prime} \mid j, j^{\prime}}$ delimited by the segments $\left[t_{m}, \phi^{\prime}\right]$ and $\left[t_{s}, \phi^{\prime \prime}\right]$ and the recursive calls of the procedure RoundStaircase concern the staircases disjoint from this region, we deduce that $E_{e}$ and $E_{f}$ are disjoint from the sets $E_{e^{\prime}}$ for all edges $e^{\prime}$ picked by the recursive calls of RoundStaircase to the staircase $\mathcal{S}_{i, i^{\prime} \mid j, j^{\prime}}$. Every edge of $\Gamma$ belongs to at most one staircase, therefore $E_{e} \cap E_{f}=\emptyset$ if the edges $e, f \in E$ belong to different staircases. Finally, since there are no terminals of $T$ located below or to the left of the staircase $\mathcal{S}_{i, i^{\prime} \mid j, j^{\prime}}$, no strip traverses this staircase (a strip intersecting $\mathcal{S}_{i, i^{\prime} \mid j, j^{\prime}}$ 
either coincides with $R_{i, i^{\prime}}$ and $R_{j, j^{\prime}}$, or intersects the staircase along segments of the boundary path $M_{i, j}$ ). Therefore, no edge from $E_{1}$ can be assigned to a set $E_{e}$ for some $e \in E_{2} \cap \mathcal{S}_{i, i^{\prime} \mid j, j^{\prime}}$, thus establishing (ii) and the desired inequality (3). Now, we are in position to formulate our main result:

Theorem 5.1 The rounding algorithm described in Section 4 achieves an approximation guarantee of 2 for the minimum Manhattan network problem.

Remark 1. The running time of our rounding algorithm is dominated by the time taken to solve the linear program $\left(2^{\prime}\right)$. The number of variables $f_{e}^{i, j}$ introduced for a given edge $e$ is equal to the number of rectangles $R_{i, j},\{i, j\} \in F$, to which $e$ belongs. Since each of the $O\left(n^{2}\right)$ edges of $\Gamma$ belongs to at most $O(n)$ such rectangles, we obtain that the number of variables in $\left(2^{\prime}\right)$ is $O\left(n^{3}\right)$. A similar analysis shows that the number of constraints is also $O\left(n^{3}\right)$. Therefore, the linear program $\left(2^{\prime}\right)$ can be solved in strongly polynomial time by using the algorithm of Tardos [10].

Remark 2. Given a staircase $\mathcal{S}_{i, i^{\prime} \mid j, j^{\prime}}$ and the paths $P_{i, i^{\prime}}^{+}$and $P_{j, j^{\prime}}^{+}$, the problem of constructing a minimum rectilinear network such that every terminal of $T_{i, j}$ can be connected by an $l_{1}$-path to $P_{i, i^{\prime}}^{+} \cup P_{j, j^{\prime}}^{+}$can be solved in polynomial time using dynamic programming (for example, by adapting the algorithm from [9] for the Rectilinear Steiner Arborescence problem on staircases). However, we do not know how to analyze this solution via linear programming. Furthermore, we do not have examples of staircases having an integrality gap in $\left(1^{\prime}\right)$.

Remark 3. Fig. 9 illustrates the run of the algorithm on the example with 8 terminals given in Fig. 4 (recall, this is one of the smallest instances having an integrality gap). Namely, we round up the optimal fractional solution shown in Fig. 4 and Fig.9(a). Phase 1 returns the rectilinear network drawn in Fig. 9(b). To perform Phase 2, notice that there are three non-empty staircases, each having a single terminal (in each case, this is the terminal $t_{3}$ ). The resulting Manhattan network is given in Fig. 9(c). Its length is 29, while an optimal Manhattan network has length 28.

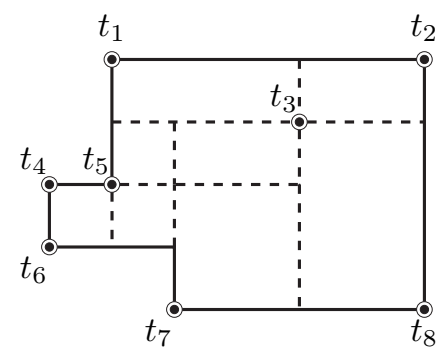

(a) Fractional optimum

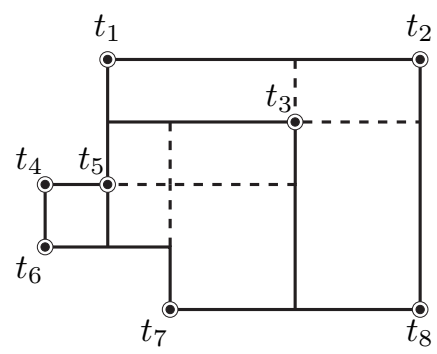

(b) After Phase 1

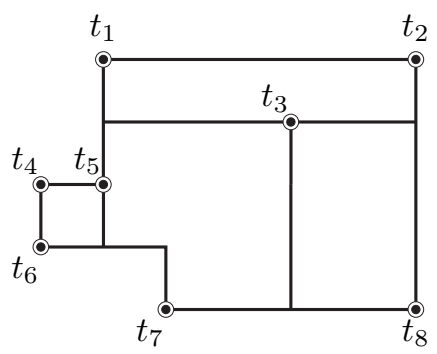

(c) Final solution

Figure 9: Example 


\section{Conclusions and perspectives}

In this paper, we presented a simple rounding algorithm for the minimum Manhattan network problem and we established that the length of the Manhattan network returned by this algorithm is at most twice the cost of the optimal fractional solution of the MMN problem. Nevertheless, experiences show that the ratio between the costs of the solution returned by our algorithm and the optimal solution of the linear programs $\left(1^{\prime}\right)$ and $\left(2^{\prime}\right)$ is much better than 2. We do not know the worst integrality gap of (1) (the worst gap obtained by computer experiences is about 1.087). Is this gap smaller or equal than 1.5? Does there exist a gap in the case when the terminals are the origin and the corners of a staircase?

The minimum Manhattan network problem can be compared with the ( $N P$-complete) Rectilinear Steiner Arborescence problem (RSA problem) [9]. In this problem, given $n$ terminals (lying in the first quadrant), one searches for a minimum rectilinear network comprising an $l_{1}$-path between the origin of coordinates and each of the $n$ terminals (clearly, such an optimal network will be a tree). The LP-formulation for the MMN problem can be viewed as a generalization of the LP-formulation of the RSA problem given in [11]. The paper [9] presents an instance of the RSA problem having an integrality gap. To our knowledge, the worst integrality gap for this problem is also not known.

Consider now the following common generalization of the MMN and RSA problems which we call the $F$-restricted $M M N$ problem: given a set of $n$ terminals and a collection $F$ of pairs of terminals, find a minimum rectilinear network $N_{F}(T)$, such that for every pair $\left\{t_{i}, t_{j}\right\} \in F$, the network $N_{F}(T)$ contains an $l_{1}$-path between $t_{i}$ and $t_{j}$. If $(T, F)$ is a complete graph, then we obtain the MMN problem and if $(T, F)$ is a star, then we obtain the RSA problem. We can show that there exists a minimum $F$-restricted Manhattan network contained in the sub-grid of $\Gamma$ generated by all empty rectangles. Using this grid, one can view (1) and (2) as integer programming formulations for the $F$-restricted MMN problem.

Notice that the rounding algorithm presented in our paper (as well as all other approximation algorithms for the MMN or RSA problems) cannot be extended in a direct way to get an approximation algorithm for the $F$-restricted MMN problem. Developing such an algorithm seems to be an interesting question. A simple example shows that the integrality gap in this case is at least 1.5: consider the four corners of a unit square as the set $T$ of terminals, and let $F$ consists of the two pairs of opposite corners of this square. Then $x_{e}=\frac{1}{2}$ for every side $e$ of the square is an optimal solution of $\left(1^{\prime}\right)$ having cost 2, while an optimal integer solution uses three edges of the square and has cost 3.

Acknowledgments. We are grateful to one of the anonymous referees for a careful reading of an early version, numerous corrections and insightful comments. This research was supported in part by the CNRS MathSTIC project "Combinatoire et géométrie des polyèdres: convexité, géométrie discrète et intégralité". 


\section{References}

[1] M. Benkert, T. Shirabe, and A. Wolff, The minimum Manhattan network problem: approximations and exact solutions. In Proc. 20th European Workshop on Computational Geometry (EWCG'04), pp. 209-212, Sevilla, 24-26 March 2004.

[2] M. Benkert, A. Wolff, and F. Widmann, The minimum Manhattan network problem: a fast factor-3 approximation. In Proc. 8th Japanese Conf. on Discrete and Computational Geometry (JCDCG'04), pp. 85-86, Tokyo, 8-11 October 2004 (Technical Report 2004-16, Fakultät für Informatik, Universität Karlsruhe, 27 pages).

[3] G. Chalmet, L. Francis, and A. Kolen, Finding efficient solutions for rectilinear distance location problems efficiently, European J. Operations Research 6 (1981) 117-124.

[4] J. Gudmundsson, C. Levcopoulos, and G. Narasimhan, Approximating a minimum Manhattan network, Nordic J. Computing 8 (2001) 219-232 and Proc. APPROX'99, 1999, pp. 28-37.

[5] D. Eppstein, Spanning trees and spanners. In J.-R. Sack and J. Urrutia, editors, Handbook of Computational Geometry, pp. 425-461, Elsevier Science Publishers B.V. NorthHolland, Amsterdam, 2000.

[6] R. Kato, K. Imai, and T. Asano, An improved algorithm for the minimum Manhattan network problem, ISAAC'02, Lecture Notes Computer Science, vol. 2518, 2002, pp. 344356.

[7] F. Lam, M. Alexanderson, and L. Pachter, Picking alignements from (Steiner) trees, J. Computational Biology 10 (2003) 509-520.

[8] K. Nouioua, Une approche primale-duale pour le problème du réseau de Manhattan minimal, RAIRO Operations Research (submitted).

[9] S.K. Rao, P. Sadayappan, F.K. Hwang, and P.W. Shor, The rectilinear Steiner arborescence problem, Algorithmica 7 (1992) 277-288.

[10] E. Tardos, A strongly polynomial algorithm to solve combinatorial linear programs, Operations Research 34 (1986) 250-256.

[11] V. Trubin, Subclass of the Steiner problem on a plane with rectilinear metric, Cybernetics 21 (1985) 320-322.

[12] V.V. Vazirani, Approximation Algorithms, Springer-Verlag, Berlin, 2001.

[13] R.E. Wendell, A.P. Hurter, and T.J. Lowe, Efficient points in location theory, AIEE Transactions 9 (1973) 314-321.

[14] M. Zachariasen, A catalog of Hanan grid problems, Networks 38 (2001) 76-83. 